\title{
Estudo da estrutura fatorial da bateria multidimensional de inteligência infantil
}

\author{
Factorial structure study of the multidimensional \\ battery for children's intelligence
}

\author{
Patrícia Waltz SCHELINI' \\ Solange WECHSLER ${ }^{2}$
}

\begin{abstract}
Resumo
Este trabalho teve como principal objetivo o estudo da estrutura fatorial da Bateria Multidimensional de Inteligência Infantil, destinada à avaliação intelectual de crianças de sete a doze anos, por meio de algumas capacidades incluídas no Modelo Cattell-Horn-Carroll. Os resultados demonstraram que, no que diz respeito às correlações entre os testes e à análise fatorial confirmatória, parece ter havido, de maneira geral, uma concordância entre os dados obtidos e os estudos associados ao modelo teórico. Além disso, a bateria mostrou-se capaz de avaliar fundamentalmente as capacidades de inteligência cristalizada e velocidade de processamento cognitivo. A maior parte da variância dessas capacidades gerais foi explicada pelos testes destinados a mensurá-las.
\end{abstract}

Palavras-chave: análise fatorial; inteligência; modelo Cattell-Horn-Carroll; testes de inteligência de Cattell.

\begin{abstract}
The goal of this paper was the factorial structure study of the Multidimensional Battery for Children 's Intelligence, a children's intelligence test based on the Cattell-Horn-Carroll's Model abilities, that assesses children from seven to twelveyears old. The results have demonstrated consonance between these data and Cattell-Horn-Carroll's Model studies. Also, the analyses demonstrated that this instrument was able to evaluate, fundamentally, the abilities of Crystallized Intelligence and Processing Speed. These capacities variance could be explained by tests focused on their measurement.
\end{abstract}

Key words: factor analysis; intelligence; Cattell-Horn-Carroll's Model; Casttell Culture Fair Intelligencetest.

A construção de um instrumento de avaliação psicológica envolve, resumidamente, a definição dos termos e constructos a serem avaliados e a operacionalização desses constructos em itens, ou seja, a especificação das atividades ou operações necessárias para medir o constructo. A definição do constructo implica a análise de modelos teóricos capazes de elucidá-lo, de modo que a elaboração dos itens não

\section{$\boldsymbol{\nabla \nabla \nabla \nabla}$}

1 Professora, Departamento de Psicologia, Universidade Federal de São Carlos. Rodovia Washington Luís, km 235, Monjolinho, 13565- 905, São Carlos, SP, Brasil. Correspondência para/Correspondence to: P.W. SCHELINI.E-mail: <pwalsche@hotmail.com>.

2 Professora Doutora, Programa de Pós-Graduação em Psicologia, Centro de Ciências da Vida, Pontifícia Universidade Católica de Campinas. Campinas, SP, Brasil.

Os autores agradecem a Fundação de Amparo à Pesquisa do Estado de São Paulo (FAPESP). 
seja intuitiva. Sobre a definição operacional, por sua vez, se fundamenta a validade da medida (Pasquali, 1999).

No que se refere à avaliação da inteligência, a Teoria das Capacidades Cognitivas de Cattell-Horn-Carroll (McGrew \& Flanagan, 1998) vem se mostrando como um dos modelos teóricos mais adequados para originar instrumentos, uma vez que é, provavelmente, o que mais diferencia e elucida as diversas capacidades que representam a inteligência.

A Teoria das Capacidades Cognitivas de Cattell-Horn-Carroll decompõe conceitos clássicos (a capacidade verbal, por exemplo) nos seus elementos mais básicos (como o desenvolvimento da linguagem, o conhecimento léxico, a capacidade de informação geral, a capacidade de comunicação e a sensibilidade gramatical), facilitando o delineamento daquilo que deverá ser avaliado e, conseqüentemente, propor- cionando uma compreensão mais precisa das informações dos resultados dos testes.

Conforme demonstrado na Tabela 1, o Modelo das Capacidades Cognitivas de Cattell-Horn-Carroll (CHC) estabelece a existência de dez capacidades gerais, dispostas na camada II, entre elas: inteligência/raciocínio fluido (Gf), raciocínio/conhecimento quantitativo $(G q)$, inteligência/raciocínio cristalizado (Gc), memória a curto prazo (Gsm), inteligência/processamento visual $(G v)$, inteligência/processamento auditivo ( $G a)$, armazenamento e recuperação associativa a longo prazo (G/r), velocidade de processamento cognitivo $(G s)$, tempo/velocidade de decisão/reação (Gt) e leitura-escrita (Grw). Cada fator geral da camada II é formado por fatores específicos, como os exemplificados naTabela 1 e estabelecidos na camada I.

Algumas capacidades específicas relacionadas às capacidades gerais de inteligência cristalizada (Gc),

Tabela 1. Representação da teoria das capacidades cognitivas de Cattell-Horn-Carroll (CHC).

\begin{tabular}{|c|c|c|c|}
\hline \multirow{2}{*}{$\begin{array}{l}\text { Fatores gerais camada II } \\
\text { Inteligência fluida (Gf) }\end{array}$} & \multicolumn{3}{|c|}{ Exemplos de fatores específicos - camada I } \\
\hline & $\begin{array}{l}\text { - Raciocínio seqüencial geral } \\
\text { - Indução }\end{array}$ & - Raciocínio quantitativo & - Raciocínio piagetiano \\
\hline Conhecimento quantitativo (Gq) & - Conhecimento matemático & - Desempenho matemático & \\
\hline Inteligência cristalizada (Gc) & $\begin{array}{l}\text { - Desenvolvimento da } \\
\text { linguagem } \\
\text { - Conhecimento léxico }\end{array}$ & $\begin{array}{l}\text { - Informação geral } \\
\text { - Informação sobre a ciência }\end{array}$ & - Capacidade de comunicação \\
\hline Memória a curto prazo (Gsm) & - Extensão da memória & - Capacidade de aprendizagem & - Memória de trabalho \\
\hline Processamento visual (Gv) & $\begin{array}{l}\text { - Visualização } \\
\text { - Relações espaciais }\end{array}$ & - Memória visual & - Análise espacial \\
\hline Processamento auditivo $(G a)$ & $\begin{array}{l}\text { - Codificação fonética } \\
\text { - Discriminação da linguagem } \\
\text { sonora }\end{array}$ & $\begin{array}{l}\text { - Discriminação geral de sons } \\
\text { - Manutenção e avaliação do } \\
\text { ritmo }\end{array}$ & $\begin{array}{l}\text { - Discriminação da duração do } \\
\text { som }\end{array}$ \\
\hline $\begin{array}{l}\text { Armazenamento e recuperação } \\
\text { associativa a longo prazo (G/r) }\end{array}$ & $\begin{array}{l}\text { - Memória associativa } \\
\text { - Memória para significados } \\
\text { - Memória espontânea }\end{array}$ & $\begin{array}{l}\text { - Fluência de idéias } \\
\text { - Fluência de palavras }\end{array}$ & $\begin{array}{l}\text { - Fluência figural } \\
\text { - Flexibilidade figural } \\
\text { - Originalidade/criatividade }\end{array}$ \\
\hline $\begin{array}{l}\text { Velocidade de processamento } \\
\text { cognitivo (Gs) }\end{array}$ & - Velocidade perceptual & - Velocidade de resposta ao teste & - Facilidade numérica \\
\hline Velocidade de decisão (Gt) & • Tempo de reação simples & - Tempo de reação para escolha & $\begin{array}{l}\text { - Velocidade de processamento } \\
\text { semântico }\end{array}$ \\
\hline Leitura-escrita (Grw) & $\begin{array}{l}\text { - Decodificação da leitura } \\
\text { - Compreensão da leitura }\end{array}$ & $\begin{array}{l}\text { - Compreensão da linguagem } \\
\text { verbal }\end{array}$ & - Capacidade de escrita \\
\hline
\end{tabular}


inteligência fluida (Gf), conhecimento quantitativo (Gq), memória a curto prazo (Gsm), armazenamento e recuperação associativa a longo prazo (G/r) e velocidade de processamento cognitivo (Gs) foram escolhidas, dentre as demais descritas no Modelo de Cattell-Horn-Carroll, para serem avaliadas pela Bateria Multidimensional de Inteligência Infantil (BMI), instrumento elaborado por Schelini (2002) e investigado no presente estudo. Essas capacidades gerais são indicadas como as mais relacionadas às exigências escolares, especialmente no que diz respeito à leitura e à matemática (Friedman, 1995; Flanagan, Andrews \& Genshaft, 1997). A seguir são descritas as definições das capacidades específicas do Modelo Cattell-Horn-Carroll enfatizadas neste estudo.

No que se refere à capacidade específica de informação geral é possível afirmar que esteja relacionada à quantidade de conhecimentos gerais adquiridos, sendo influenciada pela extensão do vocabulário ou do conhecimento léxico (Campito, 1994; Flanagan \& Ortiz, 2001; McGrew, 1997; McGrew \& Flanagan, 1998). Assim, testes de informação geral tendem a se associar com aqueles que avaliam o vocabulário e também a compreensão da leitura (Carroll, 1993).

A extensão do vocabulário e o conhecimento do significado das palavras caracterizam a capacidade específica de conhecimento léxico (Campito, 1994; Flanagan \& Ortiz, 2001; McGrew, 1997). Apesar de o conhecimento léxico $(V L)$ ser fundamental para a compreensão da capacidade específica de desenvolvimento da linguagem (LD), há evidências de que seja possível considerá-lo como uma capacidade independente (Carroll, 1993).

A capacidade específica de desenvolvimento da linguagem pode ser definida como o desenvolvimento geral da língua nativa, referindo-se, ainda, ao entendimento de palavras, sentenças e parágrafos na verbalização, não incluindo, assim, a capacidade de leitura (Campito, 1994; Carroll, 1993; Flanagan \& Ortiz, 2001; McGrew \& Flanagan, 1998). Os testes utilizados para a compreensão do desenvolvimento da linguagem (LD) também podem estar relacionados aos que avaliam a aquisição de informações gerais ou específicas, uma vez que na investigação da capacidade de informação, freqüentemente se exige o conhecimento de vocábulos.
Além disso, a aquisição de informações gerais ou específicas tende a acompanhar a apreensão do vocabulário (Carroll, 1993).

A capacidade de indução está relacionada à descoberta das características (regra, conceito, processo, relação de causa) que governam um problema ou um conjunto de materiais (Flanagan \& Ortiz, 2001; McGrew, 1997; McGrew \& Flanagan, 1998). Dessa maneira, pode ser descrita como o desenvolvimento de regras gerais, idéias ou conceitos a partir de situações ou informações específicas (Kline, 2000).

As capacidades específicas incluídas no fator geral de conhecimento quantitativo (Gq), como a própria capacidade de desempenho matemático (A3), algumas vezes são confundidas com a capacidade específica de raciocínio quantitativo $(R Q)$, relacionada à inteligência fluida (Gf). Entretanto, Gq representa o acúmulo do conhecimento matemático adquirido, enquanto $R Q$ pode ser entendida como a capacidade de raciocinar indutiva e dedutivamente durante a tentativa de solucionar um problema matemático. Assim, Gq está presente em tarefas que envolvem o conhecimento geral de matemática, como o entendimento do significado do símbolo da raiz quadrada. Por outro lado, $R Q$ está presente, por exemplo, na solução de uma tarefa em que a pessoa deveria descobrir qual elemento completaria a seguinte série de números: 2468 (McGrew \& Flanagan, 1998).

A capacidade específica de extensão da memória, relacionada ao fator geral de memória a curto prazo (Gsm) do Modelo Cattell-Horn-Carroll, pode ser descrita como a quantidade de elementos (verbais, numéricos ou figurais) que a pessoa consegue recordar imediatamente após serem apresentados (Flanagan \& Ortiz, 2001; McGrew, 1997; McGrew \& Flanagan, 1998).

A memória associativa está relacionada à capacidade de lembrar de um elemento, ou uma parte de um par, quando a outra parte ou elemento é apresentada (Campito, 1994; Carroll, 1993; Flanagan \& Ortiz, 2001; McGrew, 1997; McGrew \& Flanagan, 1998). As variáveis relacionadas à capacidade específica de memória associativa (MA) tendem a ser comparadas com aquelas que envolvem a extensão da memória (MS). Porém, a correlação entre as duas capacidades é baixa (por volta de 0,18), o que talvez seja explicado pelo fato de que, nas tarefas que avaliam a memória 
associativa $(M A)$, um tempo maior é destinado à observação dos estímulos, em comparação às atividades que envolvem a extensão da memória (MS). O período de tempo maior é fundamental para a realização de associações que irão colaborar na fixação dos estímulos a serem recordados. Por essa razão, a memória associativa parece depender mais da memória a médio ou longo prazo do que da memória a curto prazo (Carroll, 1993; Kline, 2000).

A velocidade de resposta ao teste consiste em realizar, de maneira rápida, testes relativamente fáceis ou que exijam decisões muito simples (Campito, 1994; Carroll, 1993; Flanagan \& Ortiz, 2001; McGrew, 1997; McGrew \& Flanagan, 1998). Finalmente, a velocidade perceptual corresponde à capacidade de rapidamente procurar e comparar símbolos visuais apresentados lado a lado ou separados em um campo visual.

O presente trabalho teve como principal objetivo o estudo da estrutura fatorial da Bateria Multidimensional de Inteligência Infantil (Schelini, 2002).

\section{Método}

\section{Participantes}

A amostra de participantes foi constituída por 206 crianças de 7 a 12 anos de idade, distribuídas em 6 faixas etárias com amplitude de um ano. Cada faixa etária foi representada por uma média de 34 crianças, sendo 17 do sexo feminino e 17 do sexo masculino. Em cada faixa etária do sexo feminino, aproximadamente 14 crianças eram estudantes de escolas públicas e três de escolas particulares, o mesmo ocorrendo em relação ao sexo masculino. Colaboraram para a formação da amostra nove escolas, localizadas em seis cidades do interior do estado de São Paulo, dentre elas: Campinas, São Carlos, Indaiatuba, Itapetininga, Mogi-Mirim e Sorocaba.

\section{Material}

O material (Bateria Multidimensional de Inteligência Infantil - BMI) foi composto por nove testes destinados a avaliar algumas capacidades relativas ao Modelo Cattell-Horn-Carroll (CHC). Na Tabela 2 a seguir, os instrumentos que compõem a Bateria Multidimensional de Inteligência Infantil (BMI) são citados e resumidamente descritos.

\section{Procedimento}

Primeiramente foram definidas as escolas públicas e particulares que participaram da amostra. 0 critério adotado para a definição das escolas foi,

Tabela 2. Principais características da Bateria Multidimensional de Inteligência Infantil (BMI).

\begin{tabular}{|c|c|c|}
\hline Testes & Características & Itens \\
\hline 1) Informação geral & $\begin{array}{l}\text { Questões, apresentadas oralmente, sobre conhecimentos relativos a objetos, lugares, } \\
\text { eventos e pessoas. }\end{array}$ & $1-49$ \\
\hline 2) Memória associativa & $\begin{array}{l}\text { São apresentados doze extra-terrestres, sendo que do lado de cada um está seu respectivo } \\
\text { planeta. Em seguida é apresentado um extra-terrestre e a criança deve localizar, no meio } \\
\text { de vários planetas, qual é o planeta deste extra-terrestre. }\end{array}$ & $1-12$ \\
\hline 3) Indução & $\begin{array}{l}\text { Figuras de diferentes formas, cores e tamanhos são apresentadas de modo que a tarefa } \\
\text { da criança consiste em dizer qual o critério (tamanho, forma, cor) responsável pelo fato } \\
\text { de um grupo de figuras ser diferente do outro. }\end{array}$ & $1-40$ \\
\hline 4) Memória para nomes & $\begin{array}{l}\text { Seqüências de nomes (substantivos) que a criança deve repetir oralmente e de maneira } \\
\text { idêntica a que foram apresentadas. }\end{array}$ & $1-15$ \\
\hline 5) Desempenho em matemática & Problemas aritméticos que devem ser resolvidos e respondidos oralmente. & $1-48$ \\
\hline 6) Vocabulário geral & $\begin{array}{l}\text { Conjunto de palavras, apresentadas oralmente, cujos significados devem ser explicados } \\
\text { pela criança. }\end{array}$ & $1-52$ \\
\hline 7) Velocidade de percepção & $\begin{array}{l}\text { A criança deve assinalar quatro conjuntos de números que, idênticos ao modelo, estão } \\
\text { dispostos em uma fileira com outros conjuntos numéricos. }\end{array}$ & 72 \\
\hline 8) Vocabulário ilustrado & Ilustrações que a criança deve identificar ou nomear. & $1-93$ \\
\hline 9) Rapidez de resposta & $\begin{array}{l}\text { Duas cores (azul e vermelho) estão relacionadas a estímulos simples (lápis, carro, coração } \\
\text { e pessoa). A criança deve associar as cores aos estímulos. }\end{array}$ & 76 \\
\hline
\end{tabular}


principalmente, a localização das instituições, uma vez que houve a tentativa de selecionar escolas situadas nas mais variadas regiões das cidades. Definidas as escolas, os objetivos e as características da pesquisa foram esclarecidos aos diretores e coordenadores, sendo a eles solicitada uma autorização para a entrada na instituição. Os nomes dos alunos de sete a doze anos foram listados, sendo sorteados os 206 participantes da amostra. Um pedido de autorização aos responsáveis pelos alunos foi enviado e, depois disso, foi iniciada a coleta de dados.

Os instrumentos foram apresentados na seguinte ordem: informação geral, memória associativa, indução, memória para nomes, desempenho em matemática, vocabulário geral, velocidade de percepção, vocabulário ilustrado e rapidez de resposta. Vale dizer que todos os itens foram aplicados, não havendo interrupção de qualquer teste.

\section{Resultados}

No que diz respeito ao teste informação geral, suas maiores correlações foram com os testes vocabulário geral $(0,88)$, vocabulário ilustrado $(0,85)$ e desempenho em matemática $(0,80)$. $O$ teste memória associativa mostrou-se mais correlacionado ao desempenho em matemática $(0,53)$, ao vocabulário geral $(0,53)$, ao informação geral $(0,52)$ e ao vocabulário ilustrado $(0,51)$ (Tabela 3).
A maior correlação do teste indução foi com o desempenho em matemática $(0,59)$. Por outro lado, o teste memória para nomes obteve maiores correlações com o vocabulário geral $(0,43)$, com o desempenho em matemática $(0,44)$ e com o velocidade de percepção $(0,42)$.

O teste desempenho em matemática mostrouse mais correlacionado com o de vocabulário geral $(0,81)$, o de informação geral $(0,80)$ e o de vocabulário ilustrado $(0,74)$. As maiores correlações do teste vocabulário geral foi com os testes informação geral $(0,88)$, vocabulário ilustrado $(0,84)$ e desempenho em matemática $(0,81)$.

O teste velocidade de percepção apresentou maiores correlações com o rapidez de resposta $(0,71)$ e vice-versa. Finalmente, o teste vocabulário ilustrado obteve uma maior correlação com o informação geral $(0,85)$, com o vocabulário geral $(0,84)$ e com o desempenho em matemática $(0,74)$. Todas as correlações são significativas a um nível menor ou igual a 1,0\% (Tabela 3).

A Tabela 4 apresenta a comparação entre três modelos utilizados na análise fatorial confirmatória por meio do qui-quadrado, dos graus de liberdade e dos índices RMS, AGFI e NPI. O índice RMS informa a existência de resíduo na comparação do modelo esperado com o observado. Os índices AGFI e NPI predizem uma variável a partir de outra já estabelecida, indicando a proporção da variância que é explicada a partir do modelo.

Tabela 3. Correlações entre os testes.

\begin{tabular}{|c|c|c|c|c|c|c|c|c|c|}
\hline & $\begin{array}{l}\text { Informação } \\
\text { geral }\end{array}$ & $\begin{array}{l}\text { Memória } \\
\text { associativa }\end{array}$ & Indução & $\begin{array}{l}\text { Memória } \\
\text { para } \\
\text { nomes }\end{array}$ & $\begin{array}{c}\text { Desempenho } \\
\text { em } \\
\text { matemática }\end{array}$ & $\begin{array}{c}\text { Vocabulário } \\
\text { geral }\end{array}$ & $\begin{array}{l}\text { Velocidade de } \\
\text { percepção }\end{array}$ & $\begin{array}{l}\text { Vocabulário } \\
\text { ilustrado }\end{array}$ & $\begin{array}{l}\text { Rapidez de } \\
\text { resposta }\end{array}$ \\
\hline Informação geral & $1,00^{* *}$ & & & & & & & & \\
\hline Memória associativa & $0,52^{* *}$ & $1,00^{* *}$ & & & & & & & \\
\hline Indução & $0,43^{* *}$ & $0,27^{* *}$ & 1,00 & & & & & & \\
\hline $\begin{array}{l}\text { Memória para } \\
\text { nomes }\end{array}$ & $0,40^{* *}$ & $0,20^{* *}$ & $0,28^{* *}$ & 1,00 & & & & & \\
\hline $\begin{array}{l}\text { Desempenho em } \\
\text { matemática }\end{array}$ & $0,80^{* *}$ & $0,53^{* *}$ & $0,59^{* *}$ & $0,44^{* *}$ & 1,00 & & & & \\
\hline Vocabulário geral & $0,88^{* *}$ & $0,53^{* *}$ & $0,50^{* *}$ & $0,43^{* *}$ & $0,81^{* *}$ & 1,00 & & & \\
\hline $\begin{array}{l}\text { Velocidade de } \\
\text { percepção }\end{array}$ & $0,68^{* *}$ & $0,41^{* *}$ & $0,33^{* *}$ & $0,42^{* *}$ & $0,65^{* *}$ & $0,67^{* *}$ & 1,00 & & \\
\hline $\begin{array}{l}\text { Vocabulário } \\
\text { ilustrado }\end{array}$ & $0,85^{* *}$ & $0,51^{* *}$ & $0,45^{* *}$ & $0,37^{* *}$ & $0,74^{* *}$ & $0,84^{* *}$ & $0,59^{* *}$ & 1,00 & \\
\hline Rapidez de resposta & $0,60^{* *}$ & $0,36^{* *}$ & $0,31^{* *}$ & $0,37^{* *}$ & $0,57^{* *}$ & $0,59^{* *}$ & $0,71^{* *}$ & $0,55^{* *}$ & 1,00 \\
\hline
\end{tabular}

${ }^{*} p<0,01 ;{ }^{* *} p<0,001$. 
Tabela 4. Índices comparativos dos três Modelos da Análise Fatorial Confirmatória.

\begin{tabular}{lccc}
\hline Modelos & $\chi^{2}$ & DF & $p$ \\
\hline $1-(G c, G s, G f, G s m)$ & 21,53 & 21 & 0,42 \\
$2-(G c, G s, G f)$ & 30,42 & 24 & 0,17 \\
$3-(g)$ & 110,68 & 27 & 0,00 \\
\hline
\end{tabular}

$\mathrm{DF}=$ Graus de liberdade.

O primeiro modelo sugerido (Modelo 1) é composto por quatro fatores gerais: inteligência cristalizada (Gc), velocidade de processamento cognitivo (Gs), inteligência fluida (Gf) e memória a curto prazo (Gsm). As variáveis consideradas em relação ao fator geral de inteligência cristalizada (Gc) foram os testes informação geral, vocabulário geral e vocabulário ilustrado. O fator geral de velocidade de processamento cognitivo (Gs) foi representado pelos testes velocidade de percepção e rapidez de resposta. O fator geral de inteligência fluida (Gf) foi associado aos testes indução e desempenho em matemática. O último fator sugerido para compor o Modelo 1 foi o de memória a curto prazo (Gsm), composto pelos testes memória associativa e memória para nomes.

O Modelo 2 é composto por três fatores gerais: inteligência cristalizada (Gc), velocidade de processamento cognitivo (Gs) e inteligência fluida (Gf). Em relação à inteligência cristalizada (Gc), as variáveis consideradas foram os testes informação geral, vocabulário geral, vocabulário ilustrado, memória associativa e memória para nomes. O fator geral de velocidade de processamento cognitivo (Gs) foi representado pelos testes velocidade de percepção e rapidez de resposta. O fator geral de inteligência fluida (Gf) foi associado aos testes indução e desempenho em matemática.

n O Modelo 3 é composto por um fator geral $(g)$. As variáveis consideradas em relação ao fator geral foram os testes informação geral, vocabulário geral, vocabulário ilustrado, velocidade de percepção, rapidez de resposta, indução, desempenho em matemática, memória associativa e memória para nomes, ou seja, todos os subtestes.

A partir da Tabela 4 é possível constatar que a discrepância (diferença) entre o Modelo 3 e o Modelo 2 $\left(\chi^{2} \Delta 3-2=80,26\right)$, sendo 3 a diferença entre os graus de liberdade (DF), é significativa a $p<0,001$. Já a discrepância entre o Modelo 2 e o Modelo $1\left(\chi^{2} \Delta\right.$ 2-1 = 8,89), também sendo 3 a diferença entre os graus de liberdade (DF), é significativa a $p<0,05$. Dessa forma, o Modelo 1, composto pelos fatores Gc, Gs, Gf e Gsm, pode ser definido como o mais adequado, uma vez que a redução na discrepância é significativa.

A Figura 1 apresenta as correlações entre os testes da Bateria Multidimensional de Inteligência Infantil e os fatores gerais sugeridos no primeiro modelo da análise fatorial confirmatória assim como as correlações entre os fatores gerais.

Dentre os testes associados, no primeiro modelo da análise fatorial, ao fator geral de inteligência cristalizada (Gc), o informação geral foi o que apresentou a maior correlação (Figura 1). O teste velocidade de percepção foi o que obteve a maior correlação com o fator de velocidade de processamento cognitivo (Gs). A maior correlação com o fator geral de inteligência fluida (Gf) foi obtida pelo teste desempenho em matemática. Finalmente, o teste memória associativa foi o que obteve a maior correlação com o fator geral de memória a curto prazo (Gsm).

Os testes que obtiveram os maiores coeficientes Delta (que indicam o que sobra da variância) foram o memória para nomes, o memória associativa e o indução, indicando que esses três testes não foram tão eficazes quanto os restantes para explicar a variância de seus respectivos fatores, uma vez que o índice Delta indica o resíduo dessa variância.

Quanto às correlações entre os fatores gerais, o fator de memória a curto prazo (Gsm) foi o que obteve as maiores correlações com todos os outros fatores (GC, Gs e Gf).

\section{Discussão}

No que diz respeito às correlações entre os testes e à análise fatorial confirmatória, parece ter havido, de maneira geral, uma concordância entre os dados obtidos e os estudos associados ao modelo teórico (Cattell-Horn-Carroll) que fundamentou a elaboração da Bateria 


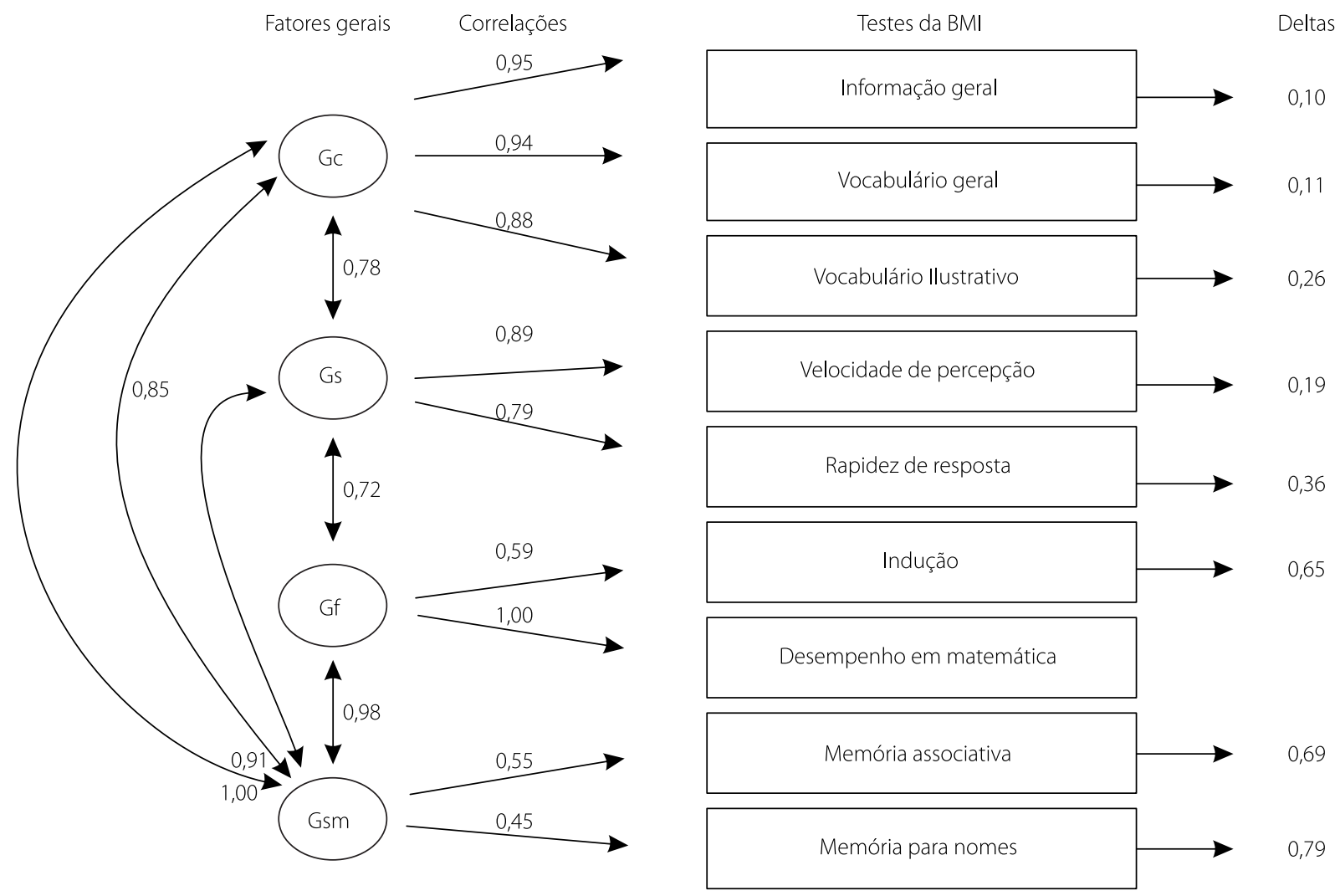

Figura 1. Correlações entre os testes da BMI e os quatro fatores gerais do Modelo 1.

Multidimensional de Inteligência Infantil (BMI). Nesse sentido, o fato de o teste informação geral ter apresentado uma maior correlação com os testes vocabulário geral e vocabulário ilustrado está de acordo com os estudos relativos às capacidades específicas que esses três instrumentos se propuseram a avaliar. A capacidade específica de informação geral (avaliada pelo teste informação geral) é descrita por vários autores (Campito, 1994; Flanagan \& Ortiz, 2001; McGrew \& Flanagan, 1998) como sendo influenciada pela capacidade específica de conhecimento léxico (avaliada pelos testes vocabulário geral e vocabulário ilustrado).

Os testes vocabulário geral e vocabulário ilustrado também foram elaborados para avaliar a capacidade específica de desenvolvimento da linguagem. Esses dois instrumentos se mostraram mais correlacionados ao teste informação geral provavelmente porque, de acordo com Carroll (1993), a aquisição de informações gerais ou específicas tende a acompanhar a apreensão do vocabulário.

As correlações elevadas e significativas entre os testes informação geral, vocabulário geral e vocabulário ilustrado parecem evidenciar que esses três testes integram um mesmo fator geral: o de inteligência cristalizada.

Os testes memória associativa e memória para nomes foram elaborados para avaliar as capacidades específicas de memória associativa e extensão da memória, respectivamente. De acordo com o Modelo Cattell-Horn-Carroll, a capacidade específica de memória associativa está relacionada à capacidade geral de armazenamento e recuperação associativa a longo prazo. A capacidade específica de extensão da memória, por outro lado, está associada à capacidade geral de memória a curto prazo. Devido à baixa correlação entre os dois testes (memória associativa e memória para nomes) e ao fato de ambos não terem 
sido capazes de explicar a maior parte da variância do fator geral de memória a curto prazo, parece haver a necessidade da elaboração de novos testes para compor, juntamente com o memória para nomes, o fator de memória a curto prazo e, com o memória associativa, o fator Glr.

No que diz respeito às correlações entre os fatores gerais, o fator de memória a curto prazo (Gsm) foi o que apresentou as maiores correlações com os outros fatores do primeiro modelo da análise fatorial confirmatória (Gc, Gs e Gf). A exigência da capacidade de memória nos vários tipos de tarefas cognitivas, conforme ressaltou Carroll (1993), talvez seja a justificativa adequada para explicar as elevadas correlações entre o fator Gsm e os demais.

O fato de o teste velocidade de percepção ter apresentado uma maior correlação com o teste rapidez de resposta (e vice-versa) parece corresponder ao modelo teórico estudado, pois as capacidades específicas de velocidade perceptual $(P)$ e velocidade de resposta ao teste (R9), avaliadas pelos dois testes, constituem o fator geral de velocidade de processamento cognitivo (Gs).

A estrutura do modelo fatorial, na qual o teste desempenho em matemática foi associado ao fator geral de inteligência fluida (Gf), mostrou-se adequada. Afinal, esse teste apresentou uma correlação máxima $(1,00)$ com Gf, indicando, provavelmente, que o desempenho em matemática poderia ser utilizado como uma medida desse fator.

As correlações entre os quatro fatores gerais (GC, Gs, Gfe Gsm) do Modelo 1 da análise fatorial confirmatória indicaram que, depois do fator geral de memória a curto prazo (Gsm), o fator com o qual inteligência fluida (Gf) obteve a maior correlação foi inteligência cristalizada (Gc). De acordo com Cattell (1987) e Cronbach (1996), as capacidades fluidas e cristalizadas estariam positivamente correlacionadas porque as cristalizadas dependeriam do investimento das capacidades fluidas na aprendizagem e a capacidade fluida utilizaria o conhecimento na formulação e verificação de hipóteses.

É possível concluir que, no que diz respeito à estrutura fatorial, a Bateria Multidimensional de Inteli- gência Infantil (BMI) mostrou-se capaz de avaliar preponderantemente dois fatores gerais: inteligência cristalizada (GC) e velocidade de processamento cognitivo (Gs). A maior parte da variância dessas capacidades gerais foi explicada pelos testes destinados a mensurá-las.

\section{Referências}

Campito, J. S. (1994). Verbal ability. In R. J. Sternberg (Ed.), Encyclopedia of human intelligence (pp.1106-1115). New York: MacMillan.

Carroll, J. B. (1993). Human cognitive abilities: a survey of factor-analytic studies. New York: Cambridge University Press.

Cattell, R. B. (1987). Intelligence: its structure, growth and action. Amsterdam: Elsevier.

Cronbach, L. J. (1996). Fundamentos da testagem psicológica (5.ed.). Porto Alegre: Artes Médicas.

Flanagan, D. P., Andrews, T. J., \& Genshaft, J. L. (1997). The functional utility of intelligence tests with special education populations. In D. P. Flanagan, J. L. Genshaft \& P. L. Harrison (Eds.), Contemporary intellectual assessment: theories, tests, and issues (pp.457-483). New York: The Guilford Press.

Flanagan, D. P., \& Ortiz, S. O. (2001). Essentials of Cross-Battery Assessment. New York: John Wiley and Sons.

Friedman, L. (1995). The space factor in mathematics: gender differences. Review of Educational Research, 65 (1), 22-50.

Kline, P. (2000). Handbook of psychological testing (2nd ed.). London: Routledge.

McGrew, K. S. (1997). Analysis of the major intelligence batteries according to a proposed comprehensive GfGc framework. In D. P. Flanagan, J. L. Genshaft \& P. L. Harrison (Eds.), Contemporary intellectual assessment: theories, tests, and issues (pp.151-179). New York: The Guilford Press.

McGrew, K. S., \& Flanagan, D. P. (1998). The intelligence test desk reference (ITDR) - Gf-Gc cross battery assessment. Boston: Allyn and Bacon.

Pasquali, L. (1999). Instrumentos psicológicos: manual prático de elaboração. Brasília: LabPAM/IBAPP.

Schelini, P. W. (2002). Bateria multidimensional de inteligência infantil: proposta de instrumento. Tese de doutorado não-publicada, Pontifícia Universidade Católica de Campinas.

Recebido em: 18//2005

Versão final reapresentada em: 22/11/2005

Aprovado em: 12/1/2005 